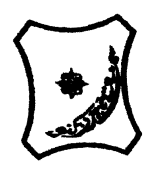

Bayero Journal of Pure and Applied Sciences, 12(1):141 - 145

Received: March, 2019

Accepted: June, 2019

ISSN $2006-6996$

\title{
ATTITUDE, PERCEPTION AND KNOWLEDGE OF MENOPAUSE BY WOMEN OFKUMANA CHIEFDOM KAURU LOCAL GOVERNMENT AREA, KADUNA STATE, NIGERIA
}

\author{
Tanko, M.; Danborno, B.; Timbuak, J. A.; Sadeeq A. A. and Bauchi, Z. M. \\ Department of Human Anatomy, Faculty of Basic Medical Science, Ahmadu Bello University, Zaria \\ Correspondence to: Tanko M., Department of Human Anatomy, A.B.U., Zaria \\ email:tankomurdakai@yahoo.com
}

Tel: +2348029314944

\begin{abstract}
Perception and experience of menopause by women is found not to be universal and wide variation occur. Majority of women are observed to lacked good knowledge of menopause, thus the negative perception toward it. The objective of the study was to determine the attitude, perception and knowledge of women in Kumana Chiefdom towards menopause. A total number of 623 women were selected randomly from the eight (8) districts in Kumana Chiefdom. Prior written/verbal consent from the women used in this study was obtained. All the subjects completed a questionnaire, which included demographic data such as age, height, weight, age at menopause, knowledge and source(s) of information on the menopause and attitude to menopause. The data was reported as Mean \pm SD (standard deviation) and percentages. Student's $t$ - test and Analysis of Variance (ANOVA) were used to test the differences in the mean of the subgroups. Chi - square tests was used to compare proportions between subgroups and according to reproductive characteristics. The result of the study showed that many women had menopausal knowledge but only few of them saw a Doctor in regard to the menopause, occupationally housewives compared to the other and the source of information on menopause to these women came from sermons in churches instead of health institutions. The perception, attitude and knowledge of women in the study population towards menopause was positive, except for the source of information which suggested that there should be improved health facilities to treat any complication that might arise due to some menopausal symptoms.
\end{abstract}

KEYWORDS; Menopause, Attitude, Perception, Knowledge, Kumana Chiefdom

\section{INTRODUCTION}

Menopause is the depletion of ovarian function that is followed by stoppage of menstruation diagnosed when a woman do not menstruate for a period of 12 consecutive months (Nusrat et al., 2008). Loss of ovarian function represents the end to fertility and onset of aging process, women who live long will experience menopause (Nusratet al., 2008). The experience of menopause by women differs and no two women experience menopause at the same time(Karimet al., 2013). Culture plays great role in the way women try to cope with menopause symptoms, although such measures are often not well documented and integrated in the public and reproductive planning systems of governments (Thomas, 2005).

Most women do not know about symptoms and health hazards of menopause, some of them don't even want to be treated of the menopausal symptoms however, few of them expressed desire to receive education about it(Leon et al 2007; Kowalcek et al., 2005; Malik, 2008; Mazhar and Erum, 2003; Noroozi et al., 2013). Numerous factors including menopausal status, social background and education, physical and emotional health may influence women's knowledge and believes about menopause (Nusrat et al., 2008).

Perception and experience of menopause by women is found not to be universal and wide variation occur due to ethnic origin (Karim et al., 2013). Many women have positive attitude towards menopause and they believed that it is a normal phase of life where they view it to be a good experience (Biri et al., 2005; Louftyet al., 2006; Pan et al., 2002). 
BAJOPAS Volume 12 Number 1, June, 2019

Studies conducted by Karim et al. (2013) discovered that women in developing countries face menopause better than the women in the western countries and that majority of the women lacked good knowledge of it according to (Adewuyi and Akinade 2010). Menopause is associated with natural progressive alterations in the hormonal production and is not an illness (Ramakuela et al., 2012) and the symptoms being an indication of the woman getting old, as such some women do not see it as an overwhelming challenge. Some women commonly view menopause as freedom from monthly bleeding, and feeling of being complete women (Ramakuelaet al., 2012) while some women see it as point of not having a child. Culture also play a role in the way women to cope with menopausal symptoms (Thomas, 2007 and Ande, 2011).

Menopause has a comprehensive effect on all body systems, experience of the symptoms vary from mild, moderate and severe, although some women do not even experience any symptoms (Ayranciet al 2010; Bauld and Brown, 2009). Both educated and uneducated women are discovered to be aware of menopause and its implication (Baig and Karim, 2006; Mustafa and Sabir, 2012; Ozumbaret al 2004). Awareness of risk factors of menopause is critical to the knowledge and attitude of women to menopause(Ayers et al., 2010). However, many of the women see the symptoms of the menopause as just something every woman has to go through in her life time and therefore the need of creating avenue to educating women on the menopause can never be underestimated. The objectives of this studies was to determine the attitude, perception and knowledge of women in Kumana Chiefdom towards menopause

\section{MATERIALS AND METHODOLOGY}

A total number of 623 women were selected randomly from eight (8) districts in Kumana Chiefdom. Prior written/verbal consent from the women used for this study was obtained. All subjects completed a questionnaire that included demographic data such as age, height(m), weight $(\mathrm{kg})$ and their reproductive history including(whether they have the knowledge menopause, their source of the menopausal knowledge and their reaction on the knowledge of the menopause). Other information contained in the questionnaire filled by the women were; educational and occupational status. Exclusion criteria included those women that refuse to complete the questionnaire, pregnant women, and those that gave incomplete or incorrect answers. The data was reported as Mean \pm SD (standard deviation) and percentages. Student's t- test and Analysis of Variance (ANOVA) were used to test the differences in the mean of the subgroups. Chi - square test was used to compare proportions between subgroups and according to reproductive characteristics. Alpha value was set as $5 \%$ and SPSS17 for Windows (IBM, Coop, NY) was used for the statistical analysis. Charts were produced using Microsoft Excel for Windows ${ }^{\circledR}$



\section{Menopausal status}

Figure 1: Graph of percentage against the menopausal statusp $\leq 0.05$ which showed that about $60 \%$ were pre-menopausal, $20 \%$ were post-menopausal, only about $10 \%$ were observed to be menopausal 


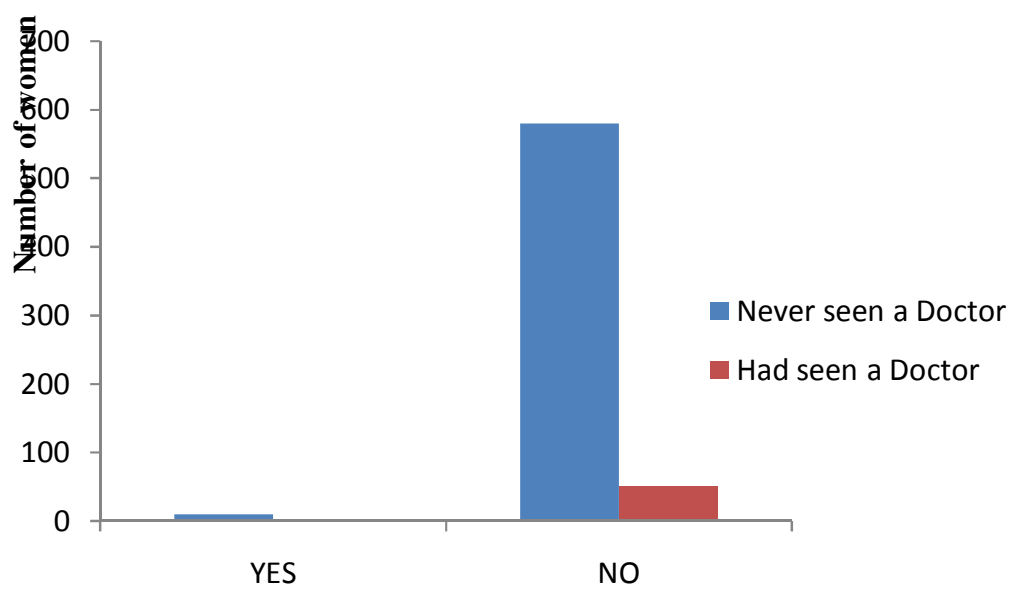

Knowledge of menopause

Figure 2: Knowledge of menopause and seeing a doctor by women of Kumana Chiefdom, $p<0.335$. The result showed that only few of the women that had the knowledge menopause had seen a Doctor concerning the menopause.

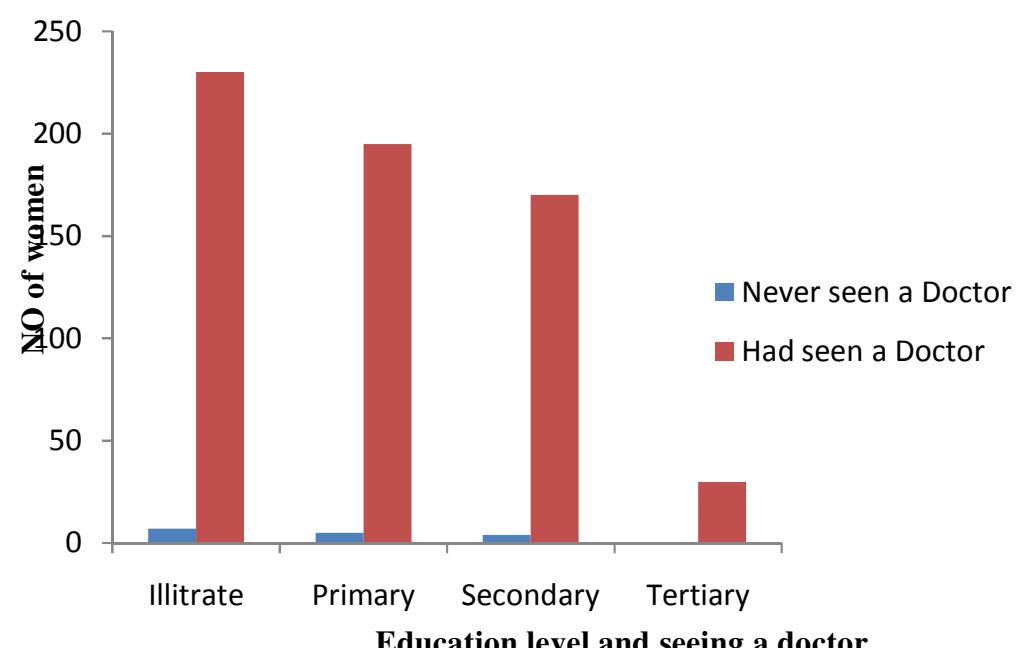

Figure3: Level of education and seeing a doctor concerning menopause of women in Kumana Chiefdom, $p<0.78$. Showed that more illiterate women had seen a Doctor concerning the menopause than those women that had some level of education.

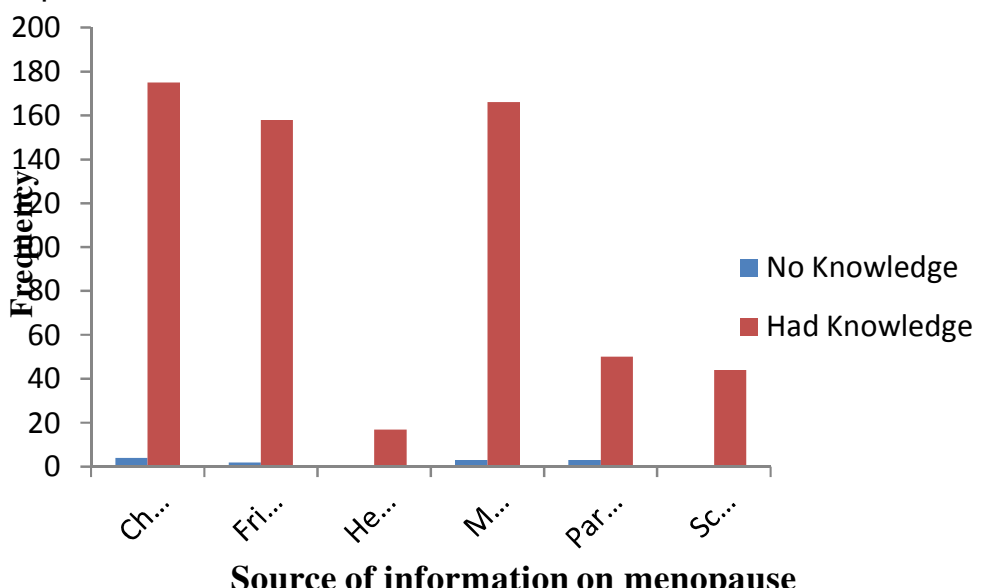

Figure 4: Source of information of menopausal knowledge of women in KumanaChiefdom, $p<0.225$. Showed that more of the women obtained knowledge of menopause from Clergy. 


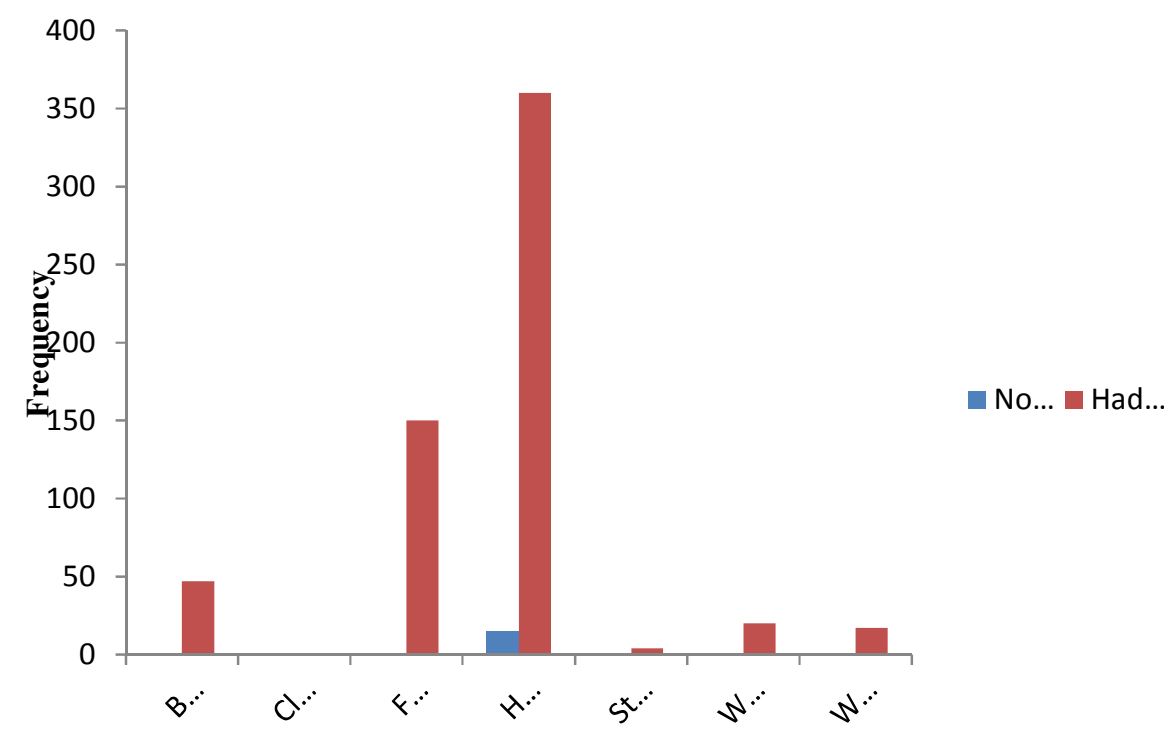

Occupational status

$\mathrm{H} /$ wives = Housewives; W/class = Working class; Wid. = Widows

Figure 5: Relationship between occupation of the women and knowledge of menopause, $p<0.515$. Showed more of the women that had knowledge of menopause that were housewives

\section{DISCUSSION}

The experience of menopause by women differs, and no two women will ever experience menopause at the same time. (Karimet al.2013) discovered that most women do not know about symptoms and health hazards of menopause and some of them do not even want to be treated of the menopausal symptoms, few expressed desire to receive education about it(Leon et a/ 2007; Kowalcek et al., 2005; Malik, 2008; Mazhar and Erum, 2003; Norooziet al., 2013).

Perception and experience of menopause by women is found not to be universal and wide variation occur due to ethnic origin (Karim et al., 2013). This study discovered that $10 \%$ of the women in this study area were menopausal, $20 \%$ post-menopausal and $70 \%$ were premenopausal, which showed that majority of the women have high reproductive success. More women have positive attitude towards menopause and believe that it is a normal phase of life, where they view it to be a good experience (Biri et al.,2002; Louftyet al.,2006; Pan et al., 2002).

In this study, many of the women were observed to have the knowledge of menopause, which was in agreement withreport of (Karim et al., 2013) and out of these women only few of them were observed to have seen a Legal practitioner concerning menopausal problems, because they considered it to be a natural phenomenon, this wasin agreement to reports of(Leon et al.,2007; Malik, 2008;Mazhar and Erum, 2003), but this could also be attributed to poor medical facilities and more of the women were observed to be educationally disadvantaged. The influence of level of education on seeing a Doctor concerning menopause appeared to be confusing because women that had some form of education reported to have seen a Doctor less than those that were illiterate, although both educated and uneducated women were observed to be aware of menopause and its implication, just as reported by (Baiget al., 2006; Mustafa and Sabir, 20012; Nusratet al., 2008; Ozumbaet al., 2004).

It was also observed that the source of knowledge about menopause came more from sermons in the Churches, followed by Health institutions, contrary to the findings of (Cassouet al., 2007). This might not be unconnected to the lack of adequate health facilities in the study location.

\section{CONCLUSION}

The perception, attitude and knowledge of women in the study population towards menopause was positive, except for the source of information which suggested that there should be improved health facilities so as to treat any complication that might arise due to some menopausal symptoms. 
BAJOPAS Volume 12 Number 1, June, 2019

\section{REFERENCES}

Adewuyi, T.D.O. and Akinade, E.A. (2010). Perception and attitude of Nigerian women towards menopause. Procedia Social and Behavioral Sciences, 5: 1777 $-1782$

Ande, A.B., Omu, O.P., Ande, O.O. and Olagbuji, N.B. (2011). Features and perceptions of menopausal women in Benin City, Nigeria. Annals of African Medicine,10:4

Ayers, B., Forshow, M. and Hunter, M. S. (2010). The impact of attitudes toward the menopause on women's symptom experience: A systemic review. Maturitas, 65(1), 28-36

Ayranci, U., Orsal, O., Arslan, G. and Emeksiz, D. F. (2010). Menopause status and attitudes in a Turkish midlife female: An epidemiological study.Women's Health, 10(1).

Baig, L. A., and Karim, S. A. (2006). Age at menopause, and knowledge of and attitudes to menopause, of women in Karachi, Pakistan. Menopause International,12(2), 71-74.

Bauld, R., and Brown, R, F. (2009). Stress, psychological distress, psychosocial factors, menopause symptoms and physical health in women. Maturitas62: 160-165

Biri A, Bakar C. and Maral I. (2005). Women with and without menopause over age of 40 in Turkey: consequences and treatment options, Maturitas. 50:167-76

Cassou, B., Mandereau, L., Aegerter, P., Touranchet, A. and Derriennic, F. (2007) Work related factors associated with age at natural menopause in a generation of French gainfully employed women. American Journal ofEpidemiology, 166(4): $429-438$

Nusrat N, Nishat Z, Afreen G, Aftab M and Asia N. (2008). Knowledge attitude and experience of Menopause. Journal of Ayuba Medical College 20: 56-59.

Karim, F., Ghaji, A., Ali, T., Jabbar, S. Naz, S. and Aslam, R. (2013). Women's perception toward menopause. Journal of Surgery, Pakistan (nternationalI), 18(3): 143-147

Kowalcek, I., Rotte, D., Banz, C., and Diedrich, K. (2005). Women's attitude and perceptions towards menopause in different cultures. Cross-cultural and intra-cultural comparison of premenopausal and post-menopausal women in Germany and in Papua New Guinea. Maturitas, 51(3), 227-35.

Thomas, S.E. (2005). "Menopause knowledge and attitudes of English-speaking Caribbean women: Implications for health education." Californian Journal of Health Promotion, 3(3), 167-176.

Leon, P., Chedraui, P., Hidalgo, L., and Ortiz, F. (2007). Perceptions and attitudes toward the menopause among middle aged women from Guayaquil, Ecuador. Maturitas, 57(3), 233-238.

Loutfy, I., Abdel Aziz, F., Dabbous, N. I., and Hassan, M. H. A. (2006). Women's perception and experience of menopause: a community-based study in Alexandria, Egypt. East MediterreanHealth Journal, 12(2), 93106.

Malik, H.S. (2008). Knowledge and attitude towards menopause and hormone replacement therapy (HRT) among postmenopausal women.Journal of Pakistan Medical Association. 58:164-7.

Mazhar, S.B and Erum, G.E.( 2003). Knowledge and attitude of elder women towards menopause. Journal of College Physicians Surgery, Pakistan,13:621624.

Mustafa,G.N. and Sabir, J. M. (2012). Perception and experience regarding menopause amongmenopaused women attending teaching hospital in Erbil City. Global Journal of Health Science, 4(3): 170-178

Noroozi, E., Dolatabadi, N.K., Eslami, A.A., Hassanzadeh, A. and Davari, S. (2013). Knowledge and attitude toward menopause phenomenon among women aged 40-45 years. Journal of Education and Health Promotion; 2: 25

Ozumba, B. C., Obi, S. N., and Obikili, W. P. (2004). Age, symptoms and perception of menopause among Nigerian women. Journal of Obstetrics and Gynecology India, 54(6), 575-578.

Pan, H., Wu, M., Hsu, C., Yao, B. and Huang, K. (2002). The perception of menopause among women in Taiwan. Maturitas. 41:269-74.

Ramakuela, N, J., Khoza, L, B., andAkinsola, H, A. (2012b). Rural women's understanding of the concept of menopause in Limpopo Province, South Africa. African Journal for Physical, Health Education, Recreation and Dance (2): 259-273. 